\title{
First-principles comparative study on the interlayer adhesion and shear strength of transition-metal dichalcogenides and graphene
}

\author{
Giacomo Levita, ${ }^{1,{ }^{*}}$ Elisa Molinari,,${ }^{1,2}$ Tomas Polcar, ${ }^{3,4}$ and Maria Clelia Righi ${ }^{1, \dagger}$ \\ ${ }^{1}$ Istituto Nanoscienze, CNR-Consiglio Nazionale delle Ricerche, I-41125 Modena, Italy \\ ${ }^{2}$ Dipartimento di Scienze Fisiche, Informatiche e Matematiche, Universitá di Modena e Reggio Emilia, I-41125 Modena, Italy \\ ${ }^{3}$ National Centre for Advanced Tribology, Faculty of Engineering and Environment, University of Southampton, Southampton SO17 1 BJ, UK \\ ${ }^{4}$ Department of Control Engineering, Faculty of Electrical Engineering, Czech Technical University in Prague, \\ Technickà 2, Prague 6, Czech Republic
}

(Received 12 May 2015; revised manuscript received 15 July 2015; published 31 August 2015)

\begin{abstract}
Due to their layered structure, graphene and transition-metal dichalcogenides (TMDs) are easily sheared along the basal planes. Despite a growing attention towards their use as solid lubricants, so far no head-to-head comparison has been carried out. By means of $a b$ initio modeling of a bilayer sliding motion, we show that graphene is characterized by a shallower potential energy landscape while more similarities are attained when considering the sliding forces; we propose that the calculated interfacial ideal shear strengths afford the most accurate information on the intrinsic sliding capability of layered materials. We also investigate the effect of an applied uniaxial load: in graphene, this introduces a limited increase in the sliding barrier while in TMDs it has a substantially different impact on the possible polytypes. The polytype presenting a parallel orientation of the layers $(R 0)$ bears more similarities to graphene while that with antiparallel orientation $(R 180)$ shows deep changes in the potential energy landscape and consequently a sharper increase of its sliding barrier.
\end{abstract}

DOI: 10.1103/PhysRevB.92.085434

PACS number(s): 68.35.Af, 71.15.Mb, 81.07.Bc

\section{INTRODUCTION}

Layered materials have recently attracted great interest for their wide range of applications, including tribology, where they constitute an important class of solid lubricants [1]. Solid lubricants play a decisive role in applications where liquid lubricants are not effective, such as, e.g., nanoscale devices, hence, the continuous experimental [2-6] and theoretical advances [7-13] in determining tribological performances of atomically thin sheets.

Powder lubricants such as graphite, boron nitride, and molybdenum disulfide share the common structure of stiff, strongly bound planes held together by weak interlayer forces, which is what favors their sliding capability. Transition-metal dichalcogenides (TMDs) $[14,15]$ are regarded as among the most suited compounds for application devices requiring dry or high-vacuum operating conditions. Indeed, in ultrahigh vacuum the performances of $\mathrm{MoS}_{2}$, the most common TMD, are superior to those of graphite [16], which on the contrary performs better in humid environments [17,18]. Furthermore, it was experimentally observed that TMDs can display lower friction coefficients when an external load is applied, at difference to most systems [19-21].

The mechanisms underlying the low friction exhibited by TMD materials have been investigated both by firstprinciples calculations [7-11] and classical dynamics simulations $[12,13]$. In particular, first-principles methods revealed the energy ordering and the electronic properties of the different stacking arrangements of $\mathrm{MoS}_{2}$ bilayers [22-26], allowing the construction of the full potential energy surface (PES) associated with the sliding motion [7,10,23]. Analogous PES data for graphene-on-graphene sliding are available [27],

\footnotetext{
*glevita@units.it

†mcrighi@unimore.it
}

but no direct comparison between the two systems was proposed so far.

Our aim is to provide a comparison between graphene and three selected molybdenum dichalcogenides $\left(\mathrm{MoS}_{2}, \mathrm{MoSe}_{2}\right.$, and $\mathrm{MoTe}_{2}$ ) with and without an external load perpendicular to a sliding bilayer, by means of a first-principles investigation on the interlayer adhesion and ideal shear strength. We will show that while the potential barriers for graphene sliding are lower than those for TMDs, the associated shear strengths show a narrower difference due to the different cell sizes. Interfacial shear strengths calculated from the derivatives of PES profiles [28] as well as bulk shear strength calculated by deforming unconstrained crystals to failure $[29,30]$ represent higher limits for the experimental values, which on the contrary include extrinsic factors such as lattice defects or mismatches, temperature, or environmental conditions [31,32]. We thus propose ideal shear strengths as valuable tools to predict the intrinsic sliding resistance of layered materials and to allow a direct comparison between the tribologic properties of commensurate, defect-free sliding systems.

A correlation with adhesive energies will be presented, along with a discussion on the different impact introduced by an applied load on the two stable TMD layer orientations. These effects are deeply entwined, as shown in our previous paper [23] and in other recent publications dealing with electronic properties of TMDs; in fact, it was shown that when subjected to a load above $20 \mathrm{GPa}, \mathrm{MoS}_{2}$ undergoes a semiconductor-to-metal transition $[24,33,34]$ as consequence of a change in stacking sequences within the bulk structure $[9,35]$. Our aim is to determine whether such a transition is associated to any change in tribologic properties. The comparison with graphene, where the transition is not reported, will also shed light on the role played by structural properties of the individual sliding layer such as size and symmetry. 


\section{COMPUTATIONAL METHODS}

We performed plane-wave, pseudopotential density functional theory (DFT) calculations [36]. A general gradient approximation (GGA) description of the exchange-correlation functional was employed by means of a dispersion-corrected Perdew-Burke-Ernzerhof (PBE-D) parametrization [37]. The contribution of van der Waals forces was included by means of the semiempirical Grimme correction [38], as done in our previous publication on $\mathrm{MoS}_{2}$ [23] and in other previous work on layered materials $[15,22,30,34]$. The scaling parameter for TMDs was set as 0.75 , while in graphene it was lowered to 0.65 as we deemed it safer to compare frictional properties by using a calculation setup reproducing correctly the experimental interlayer distances and binding energies. Although Grimme correction is not as accurate as the random phase approximation (RPA) to mimic equilibrium bulk properties [25,39], frictional properties under load (such as those investigated here) are less affected by dispersion forces $[9,40]$; furthermore, it will be shown later that the shear strength can be predicted correctly also by means of the PBE-D scheme, as it was shown that the van der Waals (vdW) contribution, although essential for a correct interlayer binding, does not significantly affect the potential corrugation [27].

The kinetic energy cutoff of the plane waves was set to 40 Ry on the basis of tests performed on the bulk structures. $A 12 \times 12 \times 4 k$-points Monkhorst-Pack grid was used to sample the TMDs bulk structures and an $18 \times 18 \times 6$ grid for graphene. In all the calculations, a Methfessel-Paxton smearing [41] of 0.01 Rydberg was employed to ease the optimization procedure and to take into account possible metallization processes at higher loads. In all systems, a hexagonal cell was used, which included one $\mathrm{MoX}_{2}$ unit per layer for the TMDs and two $\mathrm{C}$ atoms per layer for graphene. When monolayers or bilayers were modeled, the $c$ dimension of the cell was enlarged so to allow at least $12 \AA$ of vacuum between replicated images: the number of $k$ points along such direction was consequently reduced to one.

The effect of a load applied perpendicularly to the planes ( $z$ direction) was modeled by fixing the $z$ coordinate of the molybdenum atoms (in TMDs) or of one carbon atom per layer (in graphene) to form bilayers with shorter interlayer distances $d$. The load associated to such reduced interlayer spacing was evaluated as the opposite of the residual force $F_{z}$ on the fixed atoms divided by the cell area. The enthalpy of the system $H=E+P V$ was calculated as the sum of the internal energy $E$ (the total energy provided by the code) and the $P V$ contribution evaluated as the product $F_{z} d$.

The interlayer binding energy $E_{b}$ is obtained as $E_{b}=E_{12}$ $-E_{1}-E_{2}$, where $E_{12}$ is the energy of the optimized bilayer and $E_{1}$ and $E_{2}$ the energies of the single optimized layers. The work of separation $W_{\text {sep }}$, defined as the energy per unit area required to separate two layers from equilibrium to infinite distance, is calculated as $W_{\text {sep }}=E_{b} / A$, where $A$ is the unit-cell area. $W_{\text {sep }}$ can therefore be safely compared among systems having different cell sizes.

The potential corrugation is labeled as $\Delta W_{\text {sep }}$, and the lateral force $\mathbf{f}(\mathbf{r})$ experienced by a surface unit cell sliding along the direction $\mathbf{r}$ is obtained as $\mathbf{f}(\mathbf{r})=-d E_{b} / d \mathbf{r}$. The most negative value of $\mathbf{f}(\mathbf{r})$ is the maximum resistance $f_{\max }$ to sliding along the considered direction: the interfacial ideal shear strength is then $\tau(r)=-f_{\max } / A$.

\section{RESULTS AND DISCUSSION}

While graphene is a bidimensional plane of carbon atoms, each TMD layer is made up of three atomic planes, the external formed by chalcogen atoms and the internal by the metal atoms [see Fig. 1(d)]. As such, its thickness and electrostatic properties vary according to the individual composition of each TMD: for molybdenum dichalcogenides, the thickness increases going from $\mathrm{MoS}_{2}$ to $\mathrm{MoSe}_{2}$ and $\mathrm{MoTe}_{2}$, while the polarity decreases along the same sequence, following the electronegativity decrease from sulfur to tellurium.

Contrary to graphene, where two commensurate planes always show the same relative orientation, TMDs have an additional degree of freedom when forming the bilayer: the layers can be either parallel or antiparallel. In this work, we have carried out the analysis for both orientations, labeled $R 0$ and $R 180$, respectively [23]. Indeed, while most investigations on TMDs have dealt with the $R 180$ structure (typical of the $2 H$ polytype), examples of $3 R$ polytypes presenting the $R 0$ orientation were found in both natural and synthetic crystals [25,42]. Moreover, our previous publication showed that for $\mathrm{MoS}_{2}, R 0$ and $R 180$ bilayers are degenerate at zero applied load and that the degeneracy is removed by uniaxial stress [23]. We will thus limit the discussion on the zero-load behavior to the $R 180$ bilayers, and consider the $R 0$ structures when dealing with the effect of an applied load (see Supplemental Material [43]).

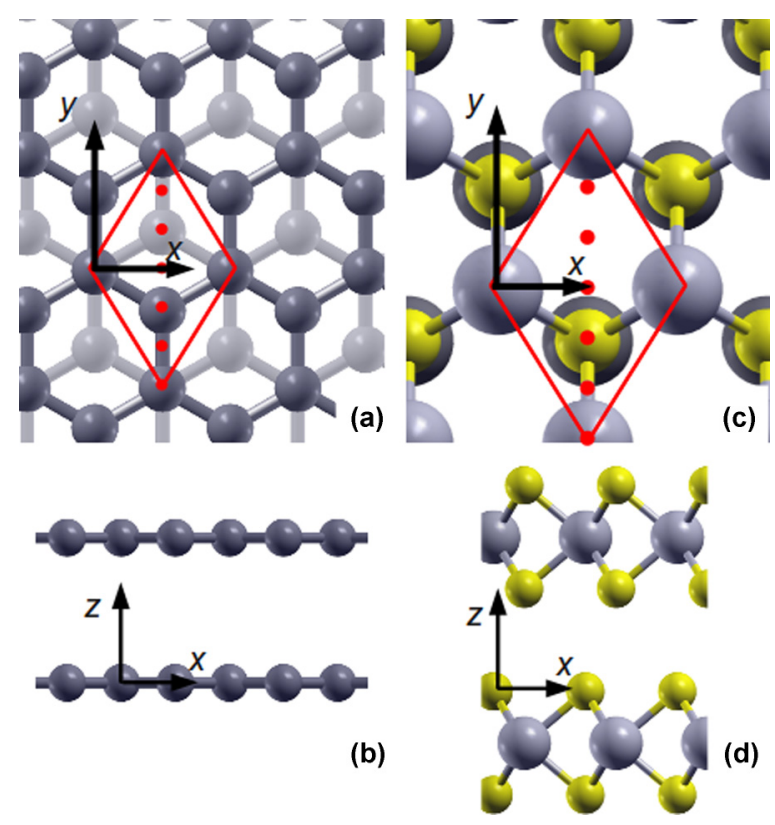

FIG. 1. (Color online) Geometry of graphene (left) and $R 180-\mathrm{MoS}_{2}$ (right) bilayers in their most stable configurations. Upper row: top views, with the unit cells marked with red solid lines [panels (a) and (c)]. Lower row: corresponding lateral views [panels (b) and (d)]. In panel (a), dark and light gray correspond to the different graphene planes. In panels (c) and (d), Mo atoms are represented in gray and $\mathrm{S}$ atoms in yellow. 
TABLE I. Calculated values for the lattice constant $(a)$, interfacial distance $\left(d_{\text {sep }}\right)$, work of separation $\left(W_{\text {sep }}\right)$, maximum sliding corrugation $\left(\Delta W_{\max }\right)$, ideal shear strengths (at $0 \mathrm{~K}$ ) along the minimum energy path $\left(\tau_{\text {mep }}\right)$ and along $y\left(\tau_{y}\right)$ for each considered bilayer system. Where available, experimental (from Refs. [44,45]) and RPA values (from Ref. [25]) are reported in parentheses for comparison.

\begin{tabular}{lcccccc}
\hline \hline & $\begin{array}{c}a \\
(\AA)\end{array}$ & $\begin{array}{c}d_{\text {sep }} \\
(\AA)\end{array}$ & $\begin{array}{c}W_{\text {sep }} \\
\left(\mathrm{J} / \mathrm{m}^{2}\right)\end{array}$ & $\begin{array}{c}\Delta W_{\text {max }} \\
\left(\mathrm{J} / \mathrm{m}^{2}\right)\end{array}$ & $\begin{array}{c}\tau_{\text {mep }} \\
(\mathrm{GPa})\end{array}$ & $\begin{array}{c}\tau_{y} \\
(\mathrm{GPa})\end{array}$ \\
\hline Graphene & 2.46 & 3.31 & 0.26 & 0.06 & 0.21 & 0.63 \\
& $(2.46)^{\mathrm{a}}$ & $(3.35)^{\mathrm{a}}$ & $(0.19)^{\mathrm{a}}$ & & $(0.14)^{\mathrm{a}}$ & \\
$\mathrm{MoS}_{2}$ & 3.19 & 3.10 & 0.27 & 0.10 & 0.50 & 0.65 \\
& $(3.16)^{\mathrm{b}}$ & $(3.08)^{\mathrm{b}}$ & $(0.30)^{\mathrm{b}}$ & $(0.10)^{\mathrm{b}}$ & $(0.16)^{\mathrm{b}}$ & \\
$\mathrm{MoSe}_{2}$ & 3.33 & 3.19 & 0.37 & 0.14 & 0.71 & 1.00 \\
$\mathrm{MoTe}_{2}$ & $(3.30)^{\mathrm{c}}$ & $(3.22)^{\mathrm{c}}$ & $(0.30)^{\mathrm{b}}$ & $(0.13)^{\mathrm{b}}$ & & \\
& 3.54 & 3.44 & 0.50 & 0.18 & 0.98 & 1.04 \\
\hline \hline
\end{tabular}

${ }^{\text {a Reference [44]. }}$

${ }^{\mathrm{b}}$ Reference [25].

${ }^{c}$ Reference [45].

\section{A. Equilibrium interlayer properties}

Table I reports the value of the lattice constant $a$ and of the optimized distance $d_{e q}$ between interfacial carbon (chalcogenide) atoms in a graphene (TMD) bilayer at zero applied load. $a$ increases monotonically from graphene to $\mathrm{MoTe}_{2}$ due to the larger dimension of the atoms; $d_{e q}$ decreases between graphene and $\mathrm{MoS}_{2}$ and increases again up to $\mathrm{MoTe}_{2}$. The works of separation $W_{\text {sep }}$ (calculated following the Methods section) are reported in Table I and show that the adhesion in graphene is only slightly lower than in $\mathrm{MoS}_{2}$, and an almost linear relation occurs between the three chalcogenides. In TMDs, the increasing size of the chalcogen atom is reflected in the increased interlayer distances and adhesion energies: a larger polarizability of the interfacial atoms leads therefore to higher van der Waals interactions between the layers. The correlation between geometric parameters and adhesive energies is shown in more detail in the Supplemental Material [43]: it must be noted that, as previously reported [27], the accuracies on sliding profile corrugations $\left(\Delta W_{\max }\right)$ are less affected by the semiempirical description of the van der Waals forces than the absolute values of the binding energies ( $\left.W_{\text {sep }}\right)$ : indeed when compared to the RPA method (which is the most reliable to model van der Waals interactions [39]) the PBE-D scheme gives for $\mathrm{MoS}_{2}$ and $\mathrm{MoSe}_{2}$ a relatively uniform error over the individual stacking orientations [25] and therefore provides a sliding corrugation very similar to that predicted by RPA. When computing the shear strengths $\left(\tau_{\text {mep }}\right)$ which are related to the derivative of such sliding potential, PBE-D and RPA yield therefore similar results.

Figure 1 highlights a line of maximum symmetry (red dots) running along the longest diagonal of the cell: such [1100] direction will be referred as $y$ henceforth. When a second layer in antiparallel orientation is placed above the first one, different bilayer geometries arise according to where the origin of the upper cell is located along the $y$ direction with respect to the lower one. The profile of the energies associated with such a translation is reported in Fig. 2. All the systems display two minima separated by two maxima: in graphene the minima are

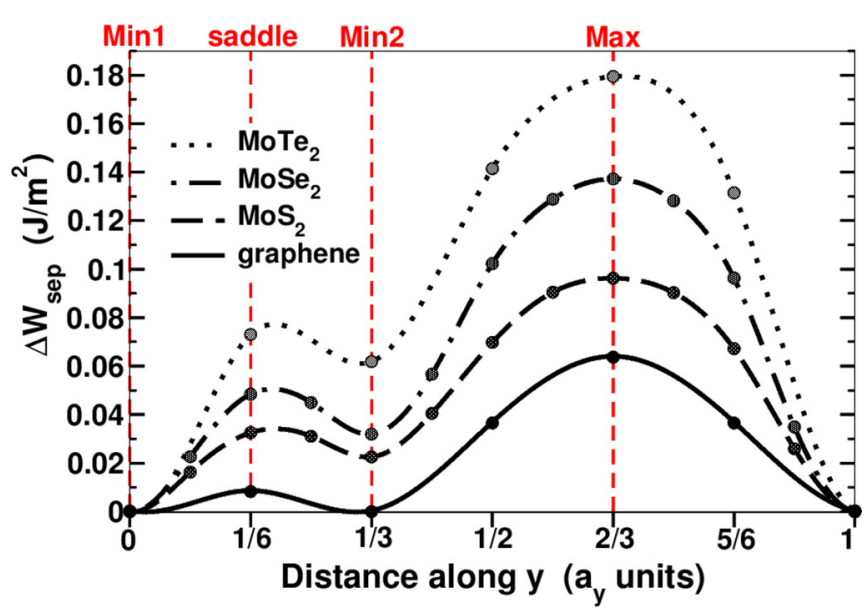

FIG. 2. (Color online) Variation of the work of separation at different bilayer configurations for each analyzed system. Each profile is associated with a bilayer sliding along the $y$ direction marked with red dots in Fig. 1. Circles correspond to the $a b$ initio data; lines are guides for the eyes only.

degenerate and correspond to the $A B$ stacking [see Fig. 1(a)], while in $R 180$ TMDs the degeneracy is removed [46]. The absolute minimum, henceforth called Min1, corresponds to the structure with the chalcogen on top of the molybdenum reported in Fig. 1(c); in the bulk this arrangement was labeled as $2 H_{c}$ [9]. The secondary minimum Min2 is obtained upon translating the top layer by $\frac{1}{3}$ along the unit cell diagonal and corresponds to the structure with on-top molybdenum atoms ( $2 \mathrm{H}_{a}$ in the bulk). The absolute maximum, which for graphene corresponds to the $A A$ stacking, in TMDs is associated with the structure having on-top chalcogen atoms at the interface; the lower maximum is a saddle point in a bidimensional representation of the energy variation (vide infra).

The heights of the absolute maxima correspond to the corrugation values reported in Table I, with an almost perfectly linear increase from graphene to $\mathrm{MoTe}_{2}$ or, in other words, from less to more interacting bilayers. Such direct correlation between sliding energy modulation and adhesion energy, while not a general rule and subject to the modelization of van der Waals interactions [25,39], has been already observed in other systems of tribological interest. In the case of TMDs, larger interfacial atoms on the one hand increase the adhesion, due to the stronger van der Waals interlayer forces; on the other, they increase the corrugation, due to the Pauli repulsion between electronic clouds of on-top chalcogen-chalcogen structures (i.e., the maxima) as it was shown that van der Waals interactions do not contribute significantly to the corrugation [27]. In the case of graphene, in fact, a much lower corrugation with respect to $\mathrm{MoS}_{2}$ is observed, despite their almost equal adhesion energies.

Considering the lattice symmetry, the energy profile along $y$ can be extended to the whole $x y$ plane: this allows to obtain the complete PES for the sliding motion. Figure 3 reports its bidimensional representation for the systems discussed so far: the length and energy color scales are common to the four panels and are indicated in the top left and right corners, respectively. In each panel, the minimum energy path (MEP) connecting the minima along the least corrugated direction 

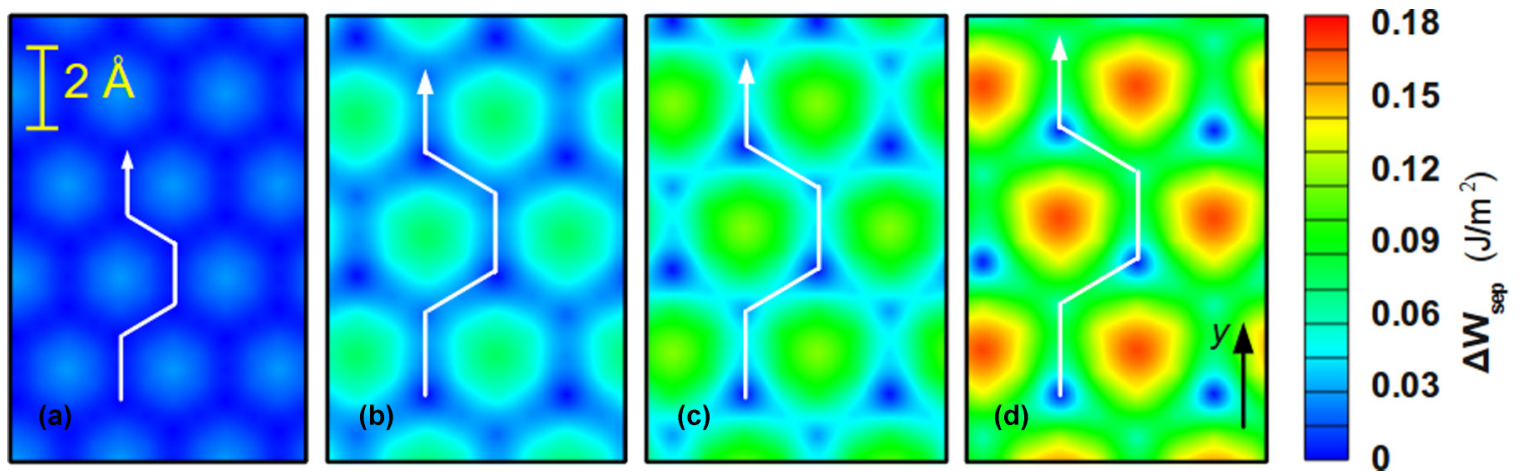

FIG. 3. (Color online) Two-dimensional maps of the potential energy surface for the sliding motion of bilayers of graphene (a), MoS $\mathrm{S}_{2}(\mathrm{~b})$, $\mathrm{MoSe}_{2}$ (c), and $\mathrm{MoTe}_{2}$ (d). The length scale is reported in the top left corner of panel (a); the orientation in the bottom right corner of panel (d). Units and color code are reported in the column on the right. In each panel MEP paths are indicated as white lines.

(i.e., through the saddle point of Fig. 2) is indicated: this forms a zigzag path characterized by angles of $60^{\circ}$. As already foreseen in Fig. 2, the PES of graphene is the smoothest and that of $\mathrm{MoTe}_{2}$ the most corrugated. However, the shorter lattice constant of graphene with respect to TMDs (see Table I) implies its higher density of stationary points within the same sliding area. This means that to cover the same distance, less dissipative events take place in TMDs than in graphene. If the kinetic friction is related to the absolute amount of dissipation occurring during the motion, it is possible that a system with higher but fewer barriers (or in other words large cell constant) can provide similar tribologic performances to one having small but frequent dissipative events. In order to investigate tribologic properties, it is therefore necessary to integrate the data on the energetic corrugation with the forces required to overcome such corrugation. $\mathrm{MoSe}_{2}$ and $\mathrm{MoTe}_{2}$ show similar tribologic properties to $\mathrm{MoS}_{2}$ and will be therefore omitted from the discussion for the sake of clarity.

Figure 4(a) displays the energy variation along the MEP profile for graphene and $\mathrm{MoS}_{2}$; contrary to Fig. 2, the horizontal axis is the actual sliding length measured in $\AA$.

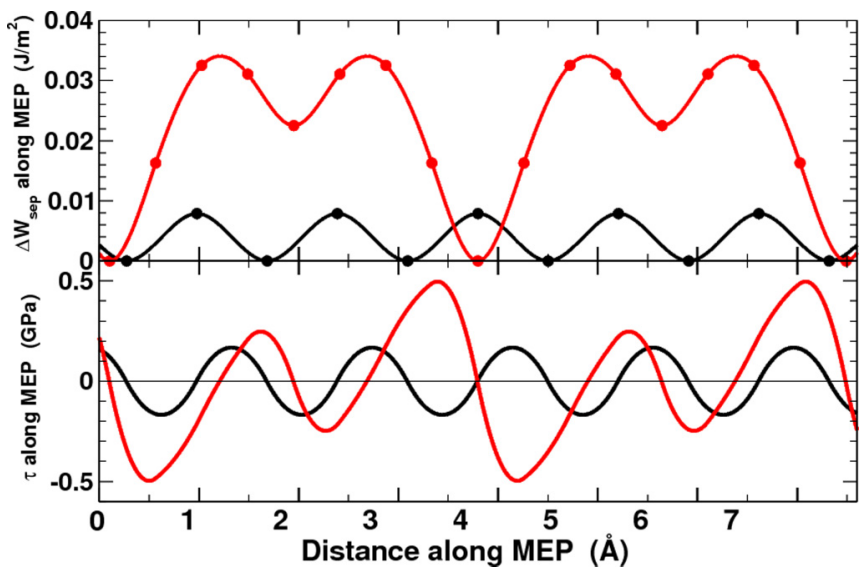

FIG. 4. (Color online) Top panel: profiles of the work of separation for graphene (black line) and $\mathrm{MoS}_{2}$ (red line) bilayers sliding along their minimum energy path (MEP). Bottom panel: the corresponding variation of the forces associated associated with the motion, normalized to the cell area. Their maximum (absolute) value corresponds to the $\tau_{\mathrm{MEP}}$ value reported in Table I.
The profile reflects the minimum-saddle-minimum paths highlighted by the zigzag lines of Fig. 3: in graphene, the minima are degenerate while in $R 180-\mathrm{MoS} 2$ the inequivalency of the minima is reflected by the volcano shape of its MEP profile. The height of the $\mathrm{MoS}_{2}$ MEP (red line) is about four times that of graphene (black line). However, its larger cell constant implies that the associated shear strength (i.e., the derivative of the work of separation curve $\tau_{\mathrm{MEP}}=-d(\Delta W$ sep $) / d$ (MEP) reported in Fig. 4(b) becomes closer to that of graphene: their ratio is in fact reduced to about 2.9. Table I reports for the four systems the values of $\tau$ calculated both along the MEP and along $y$ : these clearly point at a more similar tribological behavior than the one outlined by the barrier heights alone. It should be noted that these values only represent a theoretical upper limit for the experimental $\tau$ value, as it considers regular, defect-free bilayers at $T=0 \mathrm{~K}$. It was shown that the temperature correction alone reduces the shear strength at room temperature up to about $40 \%$ of its $0 \mathrm{~K}$ value [29]: this would bring our calculated estimate for graphene and $\mathrm{MoS}_{2}$ to roughly $85 \mathrm{MPa}$ and $0.2 \mathrm{GPa}$, respectively. A comparison with the experiments is not straightforward, as many factors (including interlayer commensurability) affect the shear strength measurements: as a result, a wide range of values are available from the literature. When a careful control over commensurate domains was carried out on graphite [44], then $\tau$ actually increased up to $0.14 \mathrm{GPa}$, which is not far from our estimate. We believe this may also apply to $\mathrm{MoS}_{2}$ where so far only values below $100 \mathrm{MPa}$ are reported [6].

Finally, as discussed in more detail in the Supplemental Material [43], we observe that for TMDs the force necessary to overcome the barrier on the maximum symmetry direction $y$ is comparable to that along the MEP, whereas in graphene such relationship of forces is much more favorable to the MEP. TMDs could therefore follow a sliding motion along a straight line rather than along the MEP: this may imply a larger distance covered by the sliding system under the same amount of dissipative events and therefore an improved tribologic performance.

\section{B. Effects of the load}

To investigate the effects of the load, we monitored the bilayer enthalpy upon compression. A compressed bilayer is 

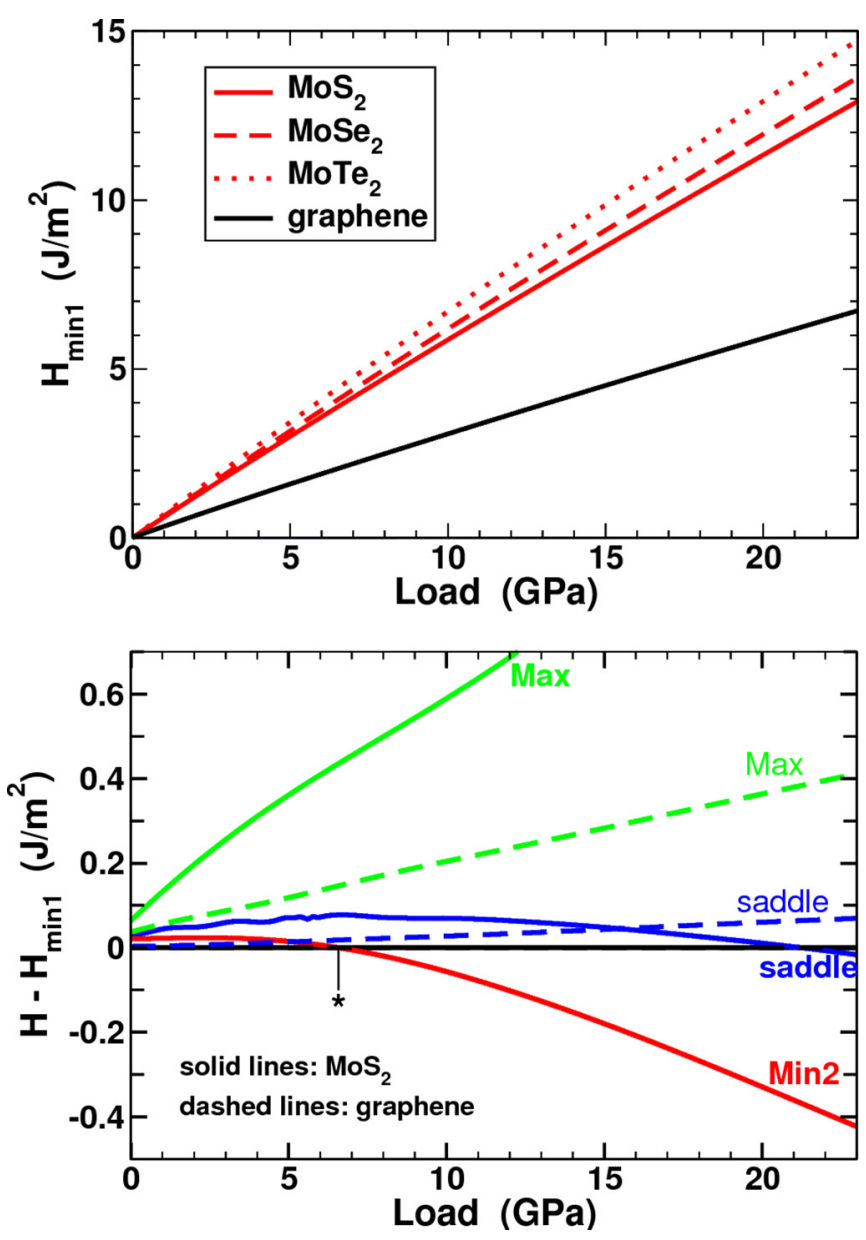

FIG. 5. (Color online) Top panel: enthalpy increase with load for the absolute minima (Min1) of graphene (black line) and TMDs (red lines) bilayers. Bottom panel: differential enthalpy behavior for selected structures on the sliding path of graphene (dashed lines) and $R 180-\mathrm{MoS}_{2}$ (solid lines) with increasing load, compared to Min1 (black baseline). For graphene, Min2 is equivalent to Min1.

simulated by fixing the $z$ coordinates of the Mo $(\mathrm{C})$ atoms in TMDs (in graphene) at shorter distances $d$ than the equilibrium one, as outlined in the Methods section. The in-plane lattice constants $a$ were left at their zero-load values, as fully relaxed calculations had shown that in $\mathrm{MoS}_{2}$ loads up to $20 \mathrm{GPa}$ introduced an in-plane strain of less than $1 \%$.

The overall effect of the load is an obvious increase of the enthalpy for all the bilayers: Figure 5(a) evidences this behavior for the absolute minima (Min1) of the four systems. The load has lower impact on graphene, while the TMDs exhibit a larger increase of enthalpy with an almost linear progression between $\mathrm{MoS}_{2}$ and $\mathrm{MoTe}_{2}$. The outcomes can be explained with the larger radius of the interfacial atoms which gives rise to a larger Pauli repulsion upon bilayer compression. Contrary to graphene, $R 180-\mathrm{TMDs}$ are characterized by nonequivalent minima which, as reported earlier $[9,23]$, are differently affected by the load. Figure 5(b) highlights this different increase by plotting the enthalpy of the PES stationary points (Min2, the saddle, and the absolute maximum) relative to the absolute minimum at zero load (Min1). In graphene (dashed lines), the enthalpies of the saddle and the maximum increase monotonically with the load (the maximum more steeply than the saddle). For $\mathrm{MoS}_{2}$ (solid lines), Min2 after an initial interval of instability becomes the most stable structure at about $7 \mathrm{GPa}$ and greatly lowers its relative energy at further increased loads. Our calculated transition pressure is in fair agreement with the semiconductor-to-metal transition pressure of 8.5 GPa obtained for a $\mathrm{MoS}_{2}$ bilayer [33].

Our present results, as well those as Singh et al. [33], show that in each investigated system the transition always occurs at lower loads for bilayers (e.g., $7 \mathrm{GPa}$ for $\mathrm{MoS}_{2}$ ) than for bulk structures (11 GPa for MoS2), indicating an increased stability of Min2 over Min1 when moving from the bulk to a bilayer (see Supplemental Material [43]). We also note that bulk $\mathrm{MoSe}_{2}$ shows no Min1-Min2 transition while bilayer $\mathrm{MoSe}_{2}$ does. The reason for such increased stability of Min2 (or rather a lower degree of destabilization with respect to Min1) is its easier compressibility: this lowers the $F d$ contribution and hence the total enthalpy despite the increase in internal energy [23]. As already reported, Min2 of $\mathrm{MoS}_{2}$ is characterized by a progressive metallization under load and by an accumulation of interfacial charge upon external compression as consequence of the formation of interlayer S-S bonds [23,24,34]: such accumulations are discussed in more detail in the Supplemental Material [43].

From a tribological point of view, the formation of this stable structure actually hampers the sliding motion at high loads, as the system will experience increasingly high barriers moving along the Min1-saddle-Min2 MEP: despite the saddle point also experiencing a stabilization over Min1, its difference with Min2 keeps on increasing with the load, especially above $10 \mathrm{GPa}$. Interlayer sliding under uniaxial pressure will then become progressively more and more difficult in $R 180-\mathrm{TMD}$ films than in graphene, which, as depicted in Fig. 5(b), presents a much smaller change in the enthalpy difference between the saddle and the absolute minimum.

It is interesting to notice that in $R 0$-TMD bilayers, where the stacked layers have parallel orientation, the degeneracy of the minima [23] is not removed by the load; their enthalpy increases at an intermediate rate between $R 180$-Min 1 and $R$ 180-Min2 (see Supplemental Material [43]). Furthermore, the saddle and the maximum are always more stable than those in $R 180$; this means that the sliding barriers increase less, and more regularly. This marks a significant difference with the $R 180$ structures where the PES changes its shape under load as a consequence of the Min1-Min2 transition.

To visually sum up the different behavior under load, Fig. 6 reports the enthalpy barrier height along the MEP paths for graphene, $R 180-\mathrm{MoS}_{2}$, and $R 0-\mathrm{MoS}_{2}$ bilayers: the other TMDs display a pattern qualitatively similar to $\mathrm{MoS}_{2}$ and are discussed in the Supplemental Material [43]. In graphene, the barrier increases faintly and monotonically; $R 0-\mathrm{MoS}_{2}$ follows a similar trend only with a slightly steeper increase. On the contrary, $R 180-\mathrm{MoS}_{2}$ presents a more complex behavior: before the Min1-Min2 transition its barrier is given by the difference between the saddle and Min1 [see Fig. 5(b)] while after that by the difference saddle-Min2. The transition pressure represents therefore a discontinuity [marked with a star in Figs. 5(b) and 6] after which the sliding barrier undergoes a significant increase. In the case of $\mathrm{MoSe}_{2}$ and $\mathrm{MoTe}_{2}$ (see Supplemental Material [43]), the onset of such 


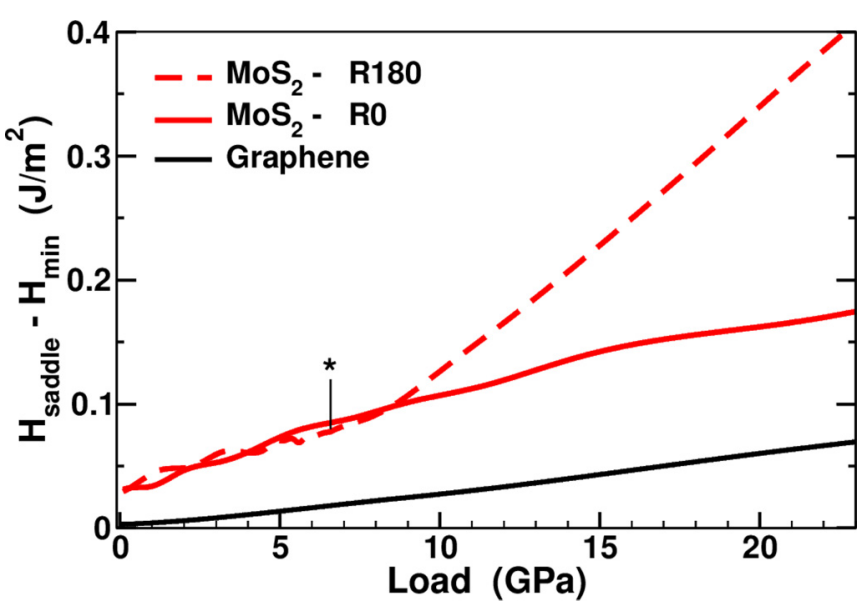

FIG. 6. (Color online) Enthalpy variation upon load for the sliding barrier associated with the MEP paths of graphene (black line) and $\mathrm{MoS}_{2}$ (red lines; solid for $R 0$, dashed for $R 180$ ), calculated at each value of the load as the difference between the saddle point and the most stable minimum structure. As in Fig. 5(b), the star marks the point where in $R 180$ Min2 becomes more stable than Min1.

transition is moved to slightly higher pressure values after which the increase of the barrier is perfectly parallel to that of $\mathrm{MoS}_{2}$. The overall consequence is that $R 180-\mathrm{Min} 2$ increasingly becomes the most stable arrangement among the whole underload TMD bilayers, but $R 0$ structures are the most easily sheared. This may infer that TMDs in the $3 R$ polytype could display better tribological performances than their $2 H$ counterparts.

Nevertheless, our results for TMDs under load never show improved intrinsic tribological performances as, instead, predicted for other van der Waals systems presenting similar changes of the PES shape induced by load [47]. We, thus, assign the experimental evidence of a friction decrease with load to other factors such as a mechanically driven formation of more regular layered domains, as observed in dynamical simulations [13] or to the Hertzian mechanical contact conditions [21]. Tribochemical reactions may also be activated by the load [48]: the experimental conditions could then become markedly different from the anhydrous, defect-free situation modeled here.

\section{CONCLUSIONS}

The comparison of intrinsic interlayer properties of molybdenum dichalcogenides and graphene, obtained by firstprinciples calculations with semiempirical inclusion of van der Waals interactions, revealed the following:

(i) In TMDs, the adhesion and corruguation of the potential energy surface for interlayer sliding increase with the size of the chalchogen atoms, reflecting a corresponding increase of the layer polarizability and the Pauli repulsion, respectively.

(ii) The potential energy surface for interlayer sliding in graphene is smoother than in TMDs, but presents a higher density of stationary points due to the smaller lattice parameter of graphene. A higher frequency of (less) dissipative events during motion is, then, expected.

(iii) The interfacial shear strength calculated from the PES derivatives is proposed as a quantitative parameter to compare the intrinsic resistance to sliding of solid interfaces in ideal conditions, i.e., in the absence of any contaminants, structural defect, or incommensurate domains. While in TMDs the shear strength does not change considerably with the direction of sliding, a much higher frictional anisotropy is predicted for graphene: The shear strength of graphene is $60 \%$ lower than that of $\mathrm{MoS}_{2}$ along the minimum energy path, while along the maximum energy path this difference decreases to $3 \%$.

Our results for the systems under uniaxial load also show the following:

(iv) In graphene, the potential energy surface preserves its shape with only a limited increase of its corrugation.

(v) In parallel TMDs layers $(R 0)$, the PES shape does not change shape under load, although the barrier increases more steeply than in graphene; on the contrary, antiparallel layers ( $R 180$ ) show a marked change in the PES shape along with a much higher increase of their barrier height. Our results, thus, point at a different frictional behavior of the $2 H$ and $3 R$ polytypes of TMDs under uniaxial pressure.

(vi) The anomalous effect of an applied load improving the tribology of $\mathrm{MoS}_{2}$ observed in some experiments should not be related to intrinsic factors governing the interlayer sliding.

\section{ACKNOWLEDGMENT}

We acknowledge the CINECA consortium for the availability of high-performance computing resources and support through the ISCRA-B "attrit0" project (2013).
[1] Scharf, S. V. Prasad, Solid lubricants: A review, J. Mater. Sci. 48, 511 (2013).

[2] C. Donnet, J.-M. Martin, Th. Le Mogne, and M. Belin, Superlow friction of $\mathrm{MoS}_{2}$ coatings in various environments, Tribol. Int. 29, 123 (1996).

[3] C. Lee, Q. Li, W. Kalb, X.-Z. Liu, H. Berger, R. W. Carpick, and J. Hone, Frictional characteristics of atomically thin sheets, Science 328, 76 (2010).

[4] K.-S. Kim, H.-J. Lee, C. Lee, S.-K. Lee, H. Jang, J.-H. Ahn, J.-H. Kim, and H.-J. Lee, Chemical vapor deposition-grown graphene: The thinnest solid lubricant, ACS Nano 5, 5107 (2011).
[5] D. Berman, A. Erdemir, and A. V. Sumant, Graphene: A new emerging lubricant, Mater. Today 17, 31 (2014).

[6] J. P. Oviedo, K. C. Santosh, N. Lu, J. Wang, K. Cho, R. M. Wallace, and M. J. Kim, In Situ TEM characterization of shearstress-induced interlayer sliding in the cross section view of molybdenum disulfide, ACS Nano 9, 1543 (2015).

[7] S. Cahangirov, C. Ataca, M. Topsakal, H. Sahin, and S. Ciraci, Frictional Figures of Merit for Single Layered Nanostructures, Phys. Rev. Lett. 108, 126103 (2012).

[8] H. Peelaers and C. G. Van de Walle, Elastic constants and pressure-induced effects in $\mathrm{MoS}_{2}$, J. Phys. Chem. C 118, 12073 (2014). 
[9] L. Hromadová, R. Martoňák, and E. Tosatti, Structure change, layer sliding, and metallization in high-pressure $\mathrm{MoS}_{2}$, Phys. Rev. B 87, 144105 (2013).

[10] T. Liang, W. G. Sawyer, S. S. Perry, S. B. Sinnott, and S. R. Phillpot, First-principles determination of static potential energy surfaces for atomic friction on $\mathrm{MoS}_{2}$ and $\mathrm{MoO}_{3}$, Phys. Rev. B 77, 104105 (2008).

[11] A. Blumberg, U. Keshet, I. Zaltsman, and O. Hod, Interlayer registry to determine the sliding potential of layered metal dichalcogenides: The Case of $2 \mathrm{H}-\mathrm{MoS}_{2}$, J. Phys. Chem. Lett. 3, 1936 (2012).

[12] T. Onodera, Y. Morita, A. Suzuki, M. Koyama, H. Tsuboi, N. Hatakeyama, A. Endou, H. Takaba, M. Kubo, F. Dassenoy, C. Minfray, L. Joly-Pottuz, J.-M. Martin, and A. Miyamoto, A computational chemistry study on friction of h-MoS 2 . Part I. mechanism of single sheet lubrication, J. Phys. Chem. B 113, 16526 (2009).

[13] M. Stefanov, A. N. Enyashin, T. Heine, and G. Seifert, Nanolubrication: How do $\mathrm{MoS}_{2}$-based nanostructures lubricate?, J. Phys. Chem. C 112, 17764 (2008).

[14] M. Chhowalla, H. S. Shin, G. Eda, L.-J. Li, K. P. Loh, and $\mathrm{H}$. Zhang, The chemistry of two-dimensional layered transition metal dichalcogenide nanosheets, Nat. Chem. 5, 263 (2013).

[15] C. Ataca, H. Sahin, and S. Ciraci, Stable, single-layer $\mathrm{MX}_{2}$ transition-metal oxides and dichalcogenides in a honeycomblike structure, J. Phys. Chem. C 116, 8983 (2012).

[16] J.-M. Martin, C. Donnet, Th. Le Mogne, and Th. Epicier, Superlubricity of molybdenum disulphide, Phys. Rev. B 48, 10583(R) (1993).

[17] D. Berman, A. Erdemir, and A. V. Sumant, Reduced wear and friction enabled by graphene layers on sliding steel surfaces in dry nitrogen, Carbon 59, 167 (2013).

[18] H. Qiu, L. Pan, Z. Yao, J. Li, Y. Shi, and X. Wang, Electrical characterization of back-gated bi-layer $\mathrm{MoS}_{2}$ field-effect transistors and the effect of ambient on their performances, Appl. Phys. Lett. 100, 123104 (2012).

[19] T. Polcar and A. Cavaleiro, Review on self-lubricant transition metal dichalcogenide nanocomposite coatings alloyed with carbon, Surf. Coat. Technol. 206, 686 (2011).

[20] J. Zekonyte, A. Cavaleiro, and T. Polcar, Frictional properties of self-adaptive chromium doped tungsten-sulfur-carbon coatings at nanoscale, Appl. Surf. Sci. 303, 381 (2014).

[21] I. L. Singer, R. N. Bolster, J. Wegand, S. Fayeulle, and B. C. Stupp, Hertzian stress contribution to low friction behavior of thin $\mathrm{MoS}_{2}$ coatings, Appl. Phys. Lett. 57, 995 (1990).

[22] Q. Liu, L. Li, Y. Li, Z. Gao, Z. Chen, and J. Lu, Tuning electronic structure of bilayer $\mathrm{MoS}_{2}$ by vertical electric field: A first-principles investigation, J. Phys. Chem. C 116, 21556 (2012).

[23] G. Levita, A. Cavaleiro, E. Molinari, T. Polcar, and M. C. Righi, Sliding properties of $\mathrm{MoS}_{2}$ layers: Load and interlayer orientation effects, J. Phys. Chem. C 118, 13809 (2014).

[24] A. P. Nayak, S. Bhattacharyya, J. Zhu, J. Liu, X. Wu, T. Pandey, C. Jin, A. K. Singh, D. Akinwande, and J.-F. Lin, Pressureinduced semiconducting to metallic transition in multilayered molybdenum disulphide, Nat. Commun. 5, 3731 (2014).

[25] J. He, K. Hummer, and C. Franchini, Stacking effects on the electronic and optical properties of bilayer transition metal dichalcogenides $\mathrm{MoS}_{2}, \mathrm{MoSe}_{2}, \mathrm{WS}_{2}$ and $\mathrm{WSe}_{2}$, Phys. Rev. B 89, 075409 (2014).

[26] T. Peng, G. Huai-Hong, Y. Teng, and Z. Zhi-Dong, Stacking stability of $\mathrm{MoS}_{2}$ bilayer: An ab initio study, Chin. Phys. B 23, 106801 (2014).

[27] M. Reguzzoni, A. Fasolino, E. Molinari, and M. C. Righi, Potential energy surface for graphene on graphene: $A b$ initio derivation, analytical description, and microscopic interpretation, Phys. Rev. B 86, 245434 (2012).

[28] G. Zilibotti and M. C. Righi, Ab Initio calculation of the adhesion and ideal shear strength of planar diamond interfaces with different atomic structure and hydrogen coverage, Langmuir 27, 6862 (2011).

[29] D. Roundy, C. R. Krenn, M. L. Cohen, and J. W. Morris, Jr., Ideal Shear Strengths of fcc Aluminum and Copper, Phys. Rev. Lett. 82, 2713 (1999).

[30] L.-p. Feng, N. Li, M.-h. Yang, and Z.-t. Liu, Effect of pressure on elastic, mechanical and electronic properties of $\mathrm{WSe}_{2}$ : A. first-principles study, Mater. Res. Bull. 50, 503 (2014).

[31] J. L. Grosseau-Poussard, P. Moine, and M. Brendle, Shear strength measurements of parallel MoSx thin films, Thin Solid Films 307, 163 (1997).

[32] P. H. Tan, W. P. Han, W. J. Zhao, Z. H. Wu, K. Chang, H. Wang, Y. F. Wang, N. Bonini, N. Marzari, N. Pugno, G. Savini, A. Lombardo, and A. C. Ferrari, The shear mode of multilayer graphene, Nat. Mater. 11, 294 (2012).

[33] S. Bhattacharyya and A. K. Singh, Semiconductor-metal transition in semiconducting bilayer sheets of transition-metal dichalcogenides, Phys. Rev. B 86, 075454 (2012).

[34] H. Guo, T. Yang, P. Tao, Y. Wang, and Z. Zhang, High pressure effect on structure, electronic structure, and thermoelectric properties of $\mathrm{MoS}_{2}$, J. Appl. Phys. 113, 013709 (2013).

[35] Z.-H. Chi, X.-M. Zhao, H. Zhang, A. F. Goncharov, S. S. Lobanov, T. Kagayama, M. Sakata, and X.-J. Chen, PressureInduced Metallization of Molybdenum Disulfide, Phys. Rev. Lett. 113, 036802 (2014).

[36] P. Giannozzi, S. Baroni, N. Bonini, M. Calandra, R. Car, C. Cavazzoni, D. Ceresoli, G. L. Chiarotti, M. Cococcioni, I. Dabo et al., QUANTUM ESPRESSO: A modular and open-source software project for quantum simulations of materials, J. Phys.: Condens. Matter 21, 395502 (2009).

[37] J. P. Perdew, M. Burke, and K. Ernzerhof, Generalized Gradient Approximation Made Simple, Phys. Rev. Lett. 77, 3865 (1996); D. Vanderbilt, Soft self-consistent pseudopotentials in a generalized eigenvalue formalism, Phys. Rev. B 41, 7892(R) (1990).

[38] S. Grimme, Semiempirical GGA-type density functional constructed with a long-range dispersion correction, J. Comput. Chem. 27, 1787 (2006); T. Bucko, J. Hafner, S. Lebegue, and J. G. Angyan, Improved description of the structure of molecular and layered crystals: Ab Initio DFT calculations with van der Waals corrections, J. Phys. Chem. A 114, 11814 (2010).

[39] T. Björkman, A. Gulans, A. V. Krasheninnikov, and R. M. Nieminen, Van der Waals bonding in layered compounds from advanced density-functional first-principles calculations, Phys. Rev. Lett. 108, 235502 (2012).

[40] M. Rifliková, R. Martoňák, and E. Tosatti, Pressure-induced gap closing and metallization of $\mathrm{MoSe}_{2}$ and $\mathrm{MoTe}_{2}$, Phys. Rev. B 90, 035108 (2014). 
[41] M. Methfessel and A. Paxton, High-precision sampling for Brillouin-zone integration in metals, Phys. Rev. B 40, 3616 (1989).

[42] W. O. Winer, Molybdenum disulfide as a lubricant: A review of the fundamental knowledge, Wear 10, 422 (1967).

[43] See Supplemental Material at http://link.aps.org/supplemental/ 10.1103/PhysRevB.92.085434 for more details on correlation between geometric parameters and potential corrugation; comparison between R180 and R0 structures in TMDs; evaluation of forces along y and along the MEP; effect of the load on the charge distribution of R180-Min2 structures; load dependence of minimum energy paths; effect of the number of layers and $\mathrm{vdW}$ forces on the Min1-Min2 inversion.

[44] Z. Liu, J. Z. Liu, Y. Cheng, Z. Li, L. Wang, and Q. Zheng, Interlayer binding energy of graphite: A mesoscopic determi- nation from deformation, Phys. Rev. B 85, 205418 (2012); Z. Liu, S.-M. Zhang, J.-R. Yang, J. Z. Liu, Y.-L. Yang, and Q.-S. Zheng, Interlayer shear strength of single crystalline graphite, Acta Mechan. Sin. 28, 978 (2012).

[45] H.-P. Komsa and A. V. Krasheninnikov, Phys. Rev. B 86, 241201(R) (2012).

[46] The $R 0$ structure has two degenerate minima as graphene: All the rest of the energetic profile is equivalent to the $R 180$ one.

[47] M. C. Righi and M. Ferrario, Pressure Induced Friction Collapse of Rare Gas Boundary Layers Sliding over Metal Surfaces, Phys. Rev. Lett. 99, 176101 (2007).

[48] G. Zilibotti, S. Corni, and M. C. Righi, Load-Induced Confinement Activates Diamond Lubrication by Water, Phys. Rev. Lett. 111, 146101 (2013). 